\title{
Low-metallicity (sub-SMC) massive stars
}

\author{
Miriam Garcia ${ }^{1}$, Artemio Herrero ${ }^{2,3}$, Francisco Najarro ${ }^{1}$, \\ Inés Camacho ${ }^{2,3}$, Daniel J. Lennon ${ }^{4}$, Miguel A. Urbaneja ${ }^{5}$ \\ and Norberto Castro ${ }^{6}$
}

\author{
${ }^{1}$ Centro de Astrobiología (INTA-CSIC), Departamento de Astrofísica. \\ Ctra. Torrejón a Ajalvir km.4, E-28850 Torrejón de Ardoz (Madrid), Spain \\ email: mgg@cab.inta-csic.es \\ ${ }^{2}$ Instituto de Astrofísica de Canarias, 38205 La Laguna (Tenerife), Spain \\ ${ }^{3}$ Departamento de Astrofísica, Universidad de La Laguna, 38206 La Laguna (Tenerife), Spain \\ ${ }^{4}$ European Space Astronomy Centre (ESA/ESAC), Villanueva de la Cañada (Madrid), Spain \\ ${ }^{5}$ Institut fuer Astro- und Teilchenphysik, Universitaet Innsbruck, Innsbruck, Austria \\ ${ }^{6}$ Astronomy Department, University of Michigan, Ann Arbor, MI 48109, USA
}

\begin{abstract}
The double distance and metallicity frontier marked by the SMC has been finally broken with the aid of powerful multi-object spectrographs installed at 8-10m class telescopes. VLT, GTC and Keck have enabled studies of massive stars in dwarf irregular galaxies of the Local Group with poorer metal-content than the SMC. The community is working to test the predictions of evolutionary models in the low-metallicity regime, set the new standard for the metal-poor high-redshift Universe, and test the extrapolation of the physics of massive stars to environments of decreasing metallicity. In this paper, we review current knowledge on this topic.
\end{abstract}

Keywords. Galaxies: individual: IC 1613, NGC 3109, WLM, Sextans A - Stars: early-type Stars: Population III - Stars: winds, outflows - Ultraviolet: stars

\section{Introduction}

Massive stars leave their imprint through the ages of the Universe and, as such, hold the key to interpret a plethora of astrophysical phenomena. The copious amount of ionizing and mechanical feedback from a population of massive stars drives locally the gas dynamics of their natal cloud, and can impact at a global level the evolution of host galaxies. Either as individuals or in binary systems, massive stars are the progenitors of the most energetic events in the Universe that can be used to probe high-redshifts: type Ibc,II supernovae (SNe) and arguably pair-instability supernovae, super-luminous supernovae and long $\gamma$-ray bursts (GRBs). The aftermath products, neutron stars and black holes, are sites of extreme physics.

In order to understand and quantify the role of massive stars in an evolving Universe, and to eventually use $\mathrm{SNe} / \mathrm{GRBs}$ as lighthouses, it is necessary to describe the variation of their physical properties as a function of metallicity. The metal-poor regime is subject to a particularly growing interest in order to understand the conditions of earlier cosmic epochs, and to ultimately extrapolate the prescriptions for physical properties to the roughly metal-free Universe at the re-ionization epoch.

We do expect significant differences between metal poor $\left(\mathrm{Z} \leqslant 1 / 10 \mathrm{Z}_{\odot}\right)$ massive stars and those in the Milky Way (MW) in terms that will translate into significant impact on their feedback. Radiation-driven winds (RDW) are ubiquitous in the hot stages of massive star evolution. They not only drive mechanical feedback but also regulate the evolution of the star (e.g. Meynet et al. 1994), and therefore the overall ionizing radiation and the final properties of the pre-SN core. Because the driving mechanism involves absorption 
Table 1. Metal-poor early-type massive stars: headcount

\begin{tabular}{|c|c|c|c|c|}
\hline | & IC 1613 & NGC 3109 & WLM & Sextans A \\
\hline $\begin{array}{l}\text { Metallicity } \\
\# \text { O-B2 stars } \\
\# \text { O-stars } \\
\# \text { Earlier than O7 }\end{array}$ & $\begin{array}{l}1 / 7 \mathrm{O}_{\odot} \\
56 \\
26 \\
4\end{array}$ & $\begin{array}{l}1 / 7 \mathrm{O}_{\odot} \\
44 \\
12 \\
0\end{array}$ & $\begin{array}{l}1 / 7 \mathrm{O} \odot \\
8 \\
2 \\
0\end{array}$ & $\begin{array}{c}1 / 10 \mathrm{Z}_{\odot} \\
12 \\
5 \\
0\end{array}$ \\
\hline
\end{tabular}

References: Azzopardi et al. (1988); Lozinskaya et al. (2002); Bresolin et al. (2006, 2007); Evans et al. (2007); Garcia \& Herrero (2013); Camacho et al. (2016), Herrero, Garcia et al. in prep.

and re-emission of photons by metallic transitions RDWs are expected to decrease with decreasing metallicity, and be almost negligible at $\mathrm{Z} \sim 1 / 1000 \mathrm{Z}_{\odot}$ except for very bright objects (Kudritzki 2002). However, the metallicity dependence of RDWs has only been confirmed observationally down to the Small Magellanic Cloud (SMC) (Mokiem et al. 2007).

The evolutionary pathways may also change drastically. Metal-poor massive stars rotating sufficiently fast may bring He produced at the stellar core to the surface, mix it in fast time-scales, and experience chemical homogeneous evolution (CHE). The star will never leave the high temperature regime of the HR-diagram (HRD); the overall ionizing energy emitted through its evolution will be enhanced with respect to analog stars that, either because of a slower initial $v_{\text {rot }}$, or a higher metallicity powered wind, do reach the red supergiant stage (RSG). For instance, a $150 \mathrm{M}_{\odot}$ undergoing $\mathrm{CHE}$ will double the amount of HI-ionizing photons and quadruple the HeII-ionizing photons with respect to the redwards evolving analog according to Szécsi et al. (2015)'s 1/50Z $\odot$ tracks. CHE of binary massive stars within the Roche lobe is one proposed scenario to generate the double $\sim 30 \mathrm{M}_{\odot}$ black hole system that produced the first detection of gravitational waves when it merged (Mandel \& de Mink 2016). Yet, while some evidence on CHE exists (Martins et al. 2013) a sufficiently large number of stars that establishes this evolutionary pathway has not been detected.

Today, the Small Magellanic Cloud stands the reference for the metal-poor Universe. Spectral libraries of SMC stars feed population synthesis models (e.g. Leitherer et al. $2001)$. However, the SMC's $1 / 5 \mathrm{Z}_{\odot}$ falls short of the $\sim 1 / 30 \mathrm{Z}_{\odot}$ metallicity at the peak of star formation (Madau \& Dickinson 2014) and is not valid an approximation for the roughly metal-free early Universe. In this paper we will review recent efforts to surpass the SMC frontier, and what we have learnt about sub-SMC metallicity massive stars.

\section{The $1 / 7 \mathrm{O}_{\odot}$ galaxies IC 1613, NGC 3109 and WLM}

The full development of the $8-10 \mathrm{~m}$ telescopes and their multi-object spectrographs (MOS) in the mid-2000's enabled the discovery and study of massive stars beyond the Magellanic Clouds with good quality data. Three galaxies were identified within reach, with promising 1/70 $\odot$ † nebular abundances: IC 1613, NGC 3109 and WLM (Fig. 1).

Spectroscopic studies with the previous generation of $4 \mathrm{~m}$ telescopes had been restricted to the brightest supergiants, LBV and WR stars, because of faint magnitude limits and crowding issues. Nowadays the $10.4 \mathrm{~m}$ Gran Telescopio Canarias (GTC) enables R 800

$\dagger$ At this point, we would like to note that metallicity $\left(\mathrm{Z} / \mathrm{Z}_{\odot}\right)$ is often calculated from the ratio of oxygen abundances against the Solar value only, with the implied hypothesis that the chemical mixture follows the Solar pattern. However, the relative ratios of elements (e.g. $\alpha / \mathrm{Fe}$ ) do vary with the chemical evolution of galaxies (as we discuss again in Sect. 2.4). For this reason, we use the notation of $\mathrm{O} / \mathrm{O}_{\odot}$ and $\mathrm{Fe} / \mathrm{Fe} \odot$ to distinguish the metallicity indicator, and only use $\mathrm{Z} / \mathrm{Z}_{\odot}$ when information from at least both $\mathrm{Fe}$ and $\mathrm{O}$ is available. 


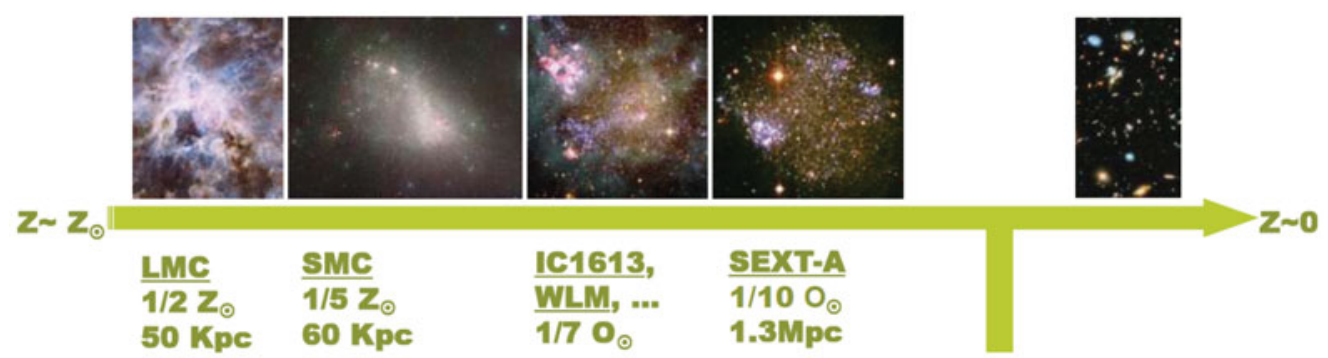

Figure 1. Road-map to the early-Universe: sequence of resolved galaxies of decreasing metal content. Characterizing massive stars in these galaxies will open the metal-poor regime. The ultimate goal is to produce prescriptions valid for the early-Universe.

identification spectroscopy of an IC 1613 O-type star at $750 \mathrm{Kpc}(\mathrm{V}=19.6)$ in 1 hour. The actual exposure times per target are being decreased with detectors of ever increasing sensitivity, and divided by the multiplexing capabilities of the MOSs.

The task first required a strong exploratory effort to confirm the nature of massive star candidates with spectroscopy. The candidates could not be directly targeted with multi-slit or multi-fiber spectrographs for deep high resolution data because blue massive stars cannot be solely identified from optical colors (though some optimized methods for the blue types exist, e.g. Garcia \& Herrero 2013). The high multi-plexing of VLT-VIMOS and FORS was of great help, although numbers are still scarce. Table 1 summarizes the number of sub-SMC metallicity early-type massive stars known to date.

Although great progress has been made, our studies are still limited in sample size, resolution and epochs. At the moment, we cannot tackle very interesting issues like rotational velocities, binary frequencies, or the possibility of a top-heavy initial mass function in metal-poor environments (in connection to the extremely massive, metal-free First Stars). However, the observations do allow for quantitative analyses to obtain stellar parameters, constrain the winds and enable first contrast against evolutionary models.

\section{1. $T_{\mathrm{eff}}$ vs Spectral Type calibration}

The effective temperatures $\left(T_{\text {eff }}\right)$ of all OBA stars studied in IC 1613, NGC 3109 and WLM are compiled in Fig. 2-left, and compared against calibrations with spectral type for various metallicities. These calibrations are useful to assign effective temperatures in sight of spectral type to first approximation, and to quantify ionizing photons emitted by unresolved populations.

The temperatures of O-stars show a large dispersion in Fig. 2-left. The scatter is of the order of the expected differences between different luminosity classes, although it likely reflects the heterogeneity of the analyzed data that either have low resolution, low signalto-noise ratio (SNR) or both. When only giant and supergiant stars are considered, the $1 / 7 \mathrm{O}_{\odot}$ effective temperature scale is roughly $\sim 1000 \mathrm{~K}$ hotter than the SMC's (Garcia \& Herrero 2013).

B-supergiants show a much smaller dispersion, and roughly follow Trundle et al. (2007)'s calibration for the SMC. The early-A supergiants can be found at the extrapolation of this calibration. One early-B supergiant stands out with high effective temperature. This is likely a miss-classification issue, since low resolution or SNR may hinder the detection of the HeII lines, which will be weak at the latest O-types.

\subsection{The HR-diagram}

The $1 / 7 \mathrm{O}_{\odot}$ massive stars are found at their expected loci in the HR-diagram (Fig. 2right). The types that have so far been confirmed by spectroscopy include O-stars and 

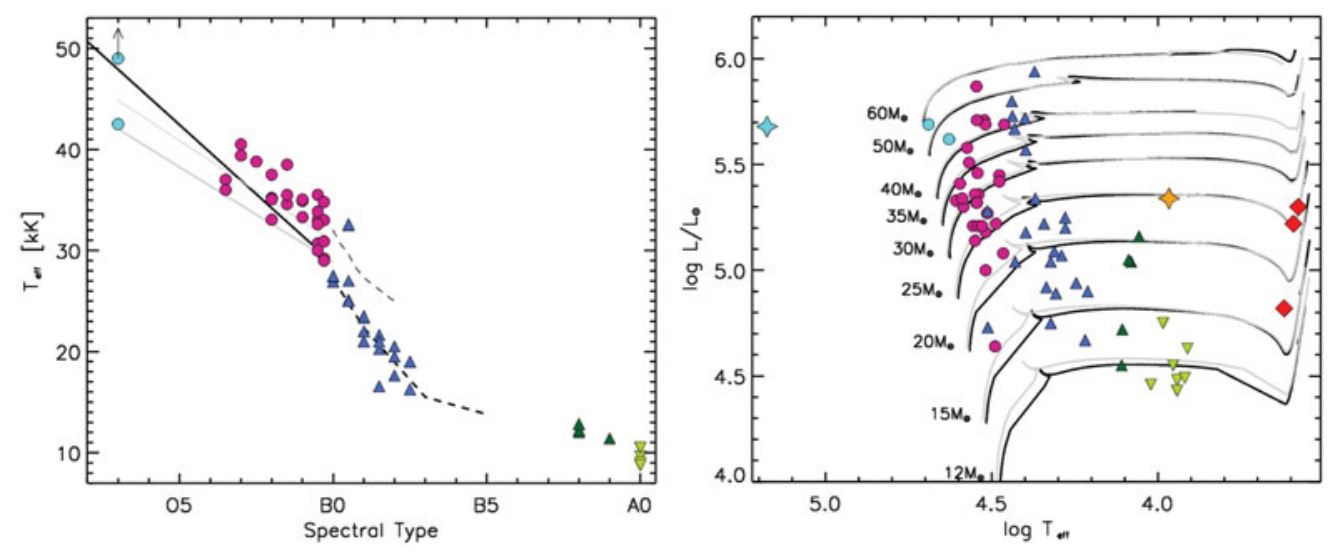

Figure 2. Properties of known stars in $1 / 7 \mathrm{O}_{\odot}$ galaxies. Left: $T_{\text {eff }} \quad$ vs spectral type for earlytype (O-A0) massive stars, compared with SMC results: Trundle et al. (2007)'s calibration for SMC B-stars (black dashed lines), and a linear fit to Mokiem et al. (2007) and Massey et al. (2009) parameters for O stars (black solid line). For comparison, the MW $T_{\text {eff }}$ vs spectral type calibration by Martins et al. (2005) is included in gray. For all calibrations: thick lines represent supergiants and thin lines dwarfs. Right: stars in the HR-diagram compared with Brott et al. (2011a)'s SMC tracks with $v_{i n i}=300 \mathrm{~km} \mathrm{~s}^{-1}$ (black) and $0 \mathrm{~km} \mathrm{~s}^{-1}$ (gray). References: Stellar samples collected from Bresolin et al. (2007); Evans et al. (2007); Tautvaišienè et al. (2007); Garcia \& Herrero (2013); Tramper et al. (2013, 2014); Hosek et al. (2014); Bouret et al. (2015); Herrero et al. (2010, 2012, in prep.), Camacho et al. in prep. Symbols: Circles: O-stars, early-types in cyan; Up-triangles: B-supergiants, late-types in dark-green; Down-triangles: A-supergiants; Red-rhomboids: RSGs; Orange-star: the LBVc V39; Cyan-star: the WO DR1.

BA supergiants, plus some advanced stages in IC 1613: RSGs, the oxygen Wolf-Rayet (WO) star DR1, and the LBV-candidate V39. O-type stars draw the main sequence, with only one early-O star close to the zero-age main sequence (ZAMS). B-supergiants overlap with the H-burning region of SMC tracks, which could be partly explained by the shift of the evolutionary paths to higher $T_{\text {eff }}$ as metallicity decreases.

The total sample is still small but at present there is no evidence of a sequence of stars undergoing CHE, which would be located at higher temperatures than the ZAMS. Likewise, no super-luminous RSG has been detected that would support the inflation and redwards evolution scenario of slowly-rotating very metal-poor stars (Szécsi et al. 2015). At the moment we cannot contrast this information with rotational velocities because of the low-resolution used for the majority of the observing runs, but no high-speed rotator has been detected.

Finally, it is noteworthy that the most massive stars detected, including the WO star in IC 1613 (Tramper et al. 2015), have $M_{i n i} \sim 60 \mathrm{M}_{\odot}$.

\subsection{The Wind Momentum - Luminosity Relation (WLR)}

Mass loss is one crucial ingredient to compute the evolution and fate of massive stars. Quantifying true mass loss rates, providing prescriptions that hold in varying metallicity environments, and testing such prescriptions in metal-poor environments, is a must to be able to simulate massive stars at high-redshifts, their death and feedback.

The WLR is currently the main diagnostic tool, and compares observational measurements of the mechanical wind-momentum $\left(\propto \dot{M} \cdot v_{\infty}\right)$ with theory. Based on the nature of RDWs, the WLR tests simultaneously the expected positive correlation of wind-momentum with stellar luminosity and its anti-correlation with metallicity. 

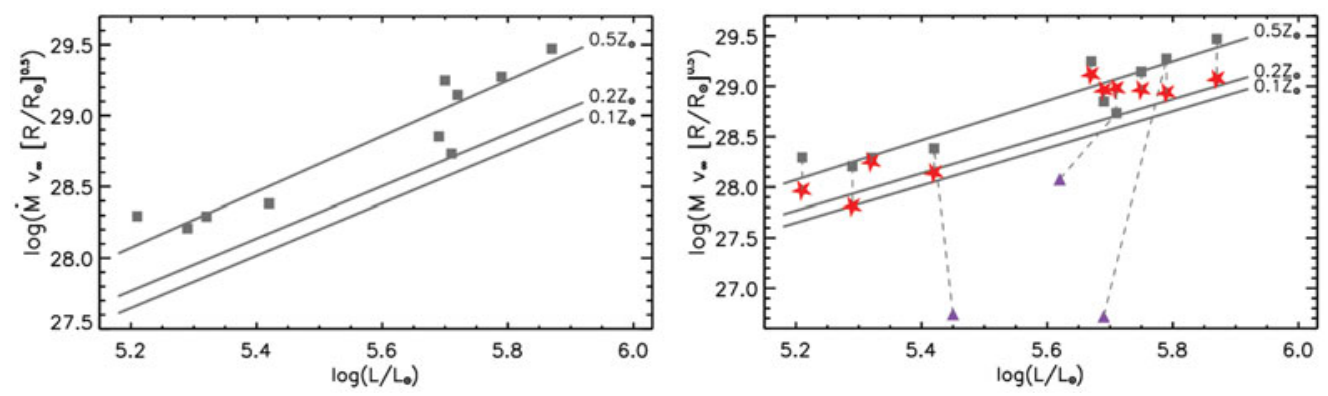

Figure 3. The $1 / 7 \mathrm{O}_{\odot}$ Wind Momentum - Luminosity Relation. Left: Wind-momentum of $1 / 7 \mathrm{O}_{\odot}$ OB-stars from optical data (Herrero et al. 2012; Tramper et al. 2011, 2014): $\dot{M}$ 's were derived from $\mathrm{H}_{\alpha}$, and $v_{\infty}=2.65 v_{\text {esc }}$ (gray squares). The unclumpled observational relations for the MW, LMC and SMC (Mokiem et al. 2007) are shown for comparison. Right: The WLR recomputed with UV terminal velocities (red stars), either direct measurements of the star or a star with similar spectral type (from Garcia et al. 2014; Bouret et al. 2015). The results from a full UV+optical analysis of 3 stars by Bouret et al. (2015) are also included (purple triangles).

When the WLR for $1 / 7 \mathrm{O}_{\odot}$ stars was first plotted, the community was alarmed: most of the stars were found at the loci of more metal-rich $0.5 \mathrm{Z}_{\odot} \quad$ LMC stars (Fig. 3left). Together with the previous detection of strong P Cygni profiles in IC 1613's V39 (Herrero et al. 2010), and the existence of a WO in the galaxy (e.g. Tramper et al. 2015), these results were suggestive of stronger winds than expected in the studied galaxies. If these results were confirmed, we would need updated wind recipes, evolutionary models, feedback calculations and SN and GRB rates for very low-metallicity massive stars.

However, the strong wind problem can be largely explained by the lack of suitable diagnostics for both the wind and metallicity (see Sect. 2.4). The optical-only studies that reported the strong winds are based on $\mathrm{H}_{\alpha}$ profile fitting. They are not sensitive to small mass loss rates and are degenerated to the parameter $\mathrm{Q}=\dot{M} /\left(v_{\infty} \cdot \mathrm{R}_{\star}\right)^{1.5}$ (Kudritzki $\&$ Puls 2000). In order to derive $\dot{M}$, and lacking a direct ultraviolet (UV) measurement, $v_{\infty}$ is calculated from the escape velocity $\left(v_{\infty} / v_{e s c}=2.65\right)$ and scaled with metallicity $\left(v_{\infty} \propto \mathrm{Z}^{0.13}\right.$ Leitherer et al. 1992). The $v_{\infty} / v_{e s c}=2.65$ calibration is long known to suffer a large scatter (Kudritzki \& Puls 2000), but recent results also argue against a straightforward $v_{\infty} \propto \mathrm{Z}$ relation (Garcia et al. 2014). Thus, in absence of UV data, $v_{\infty}$ uncertainties add up and propagate to $\dot{M}$ and the WLR.

The installation of the Cosmic Origins Spectrograph (COS) on the HST enabled UV spectroscopy $(\sim 1150-1800 \AA$ ) $)$ of OB stars out to $\sim 1 \mathrm{Mpc}$ (see Sect. 3 ), providing crucial information on their RDWs. The wind terminal velocity can be measured from the resonance lines of NV $\lambda 1238.8,1242.8$, SiIV $\lambda 1393.8,1402.8$ and CIV $\lambda 1548.2,1550.8$. Together with CIII $\lambda 1176$ and NIV $\lambda 1718.0$, these lines constrain mass loss rates even for the low $\dot{M}<10^{-7} \mathrm{M}_{\odot} \mathrm{yr}^{-1}$ values expected for metal-poor stars that render $\mathrm{H}_{\alpha}$ insensitive.

Fig. 3-right shows the $1 / 7 \mathrm{O}_{\odot}$ WLR recalculated with UV derived terminal velocities by Garcia et al. (2014) and Bouret et al. (2015). The wind-momentum of most of the stars was revised downwards, and the overall trend is now closer to Mokiem et al. (2007)'s regression describing the $\mathrm{SMC}\left(0.2 \mathrm{Z}_{\odot}\right)$. Moreover, the joint UV+optical analysis of 3 stars by Bouret et al. (2015) yielded wind-momenta much below the $1 / 7 \mathrm{Z}_{\odot}$ line. While the difference is partly due to the consideration of clumping in their study, the authors also propose the existence of a hot gas component in the wind that do not impact P Cygni profiles and therefore cannot be diagnosed from the UV. In any case, the WLR 


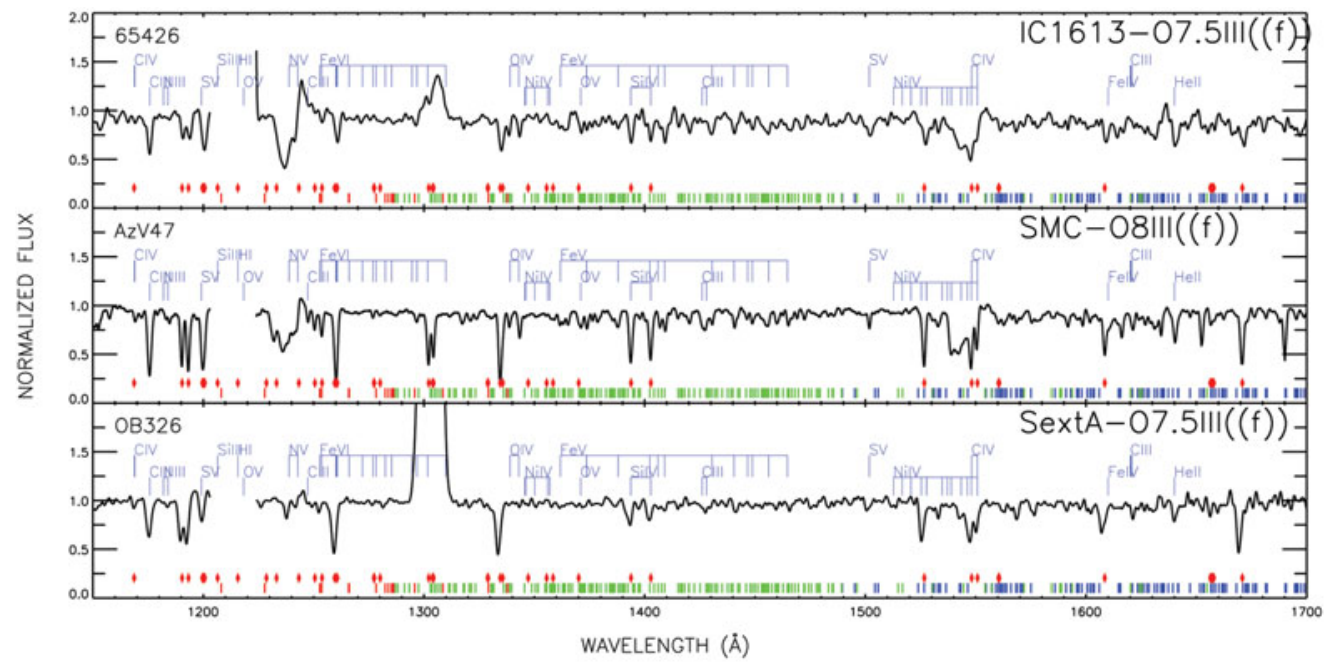

Figure 4. First UV spectroscopy at sub-SMC metallicity. The chart compares HST-COS-G140L observations of stars in IC 1613 and Sextans A (PI M. Garcia) with the STIS spectrum of a star with similar spectral type. \#65426 in IC 1613 shows the strongest P Cygni profiles of CIV and NV, stronger than the SMC star. The wind profiles are much weaker in the Sextans A star, as expected with $1 / 10 \mathrm{Z}_{\odot}$ metallicity, although the terminal velocity can still be measured from CIV. The plot also illustrates striking differences in the Fe-content; the lines in the FeV forest (green ticks) are strongest in the IC 1613 star, and decrease as we move downwards in the plot, suggesting a succession of decreasing metallicity.

of the $1 / 7 \mathrm{O}_{\odot}$ galaxies is still not settled and clearly requires a larger sample of high quality spectra.

\subsection{The actual metallicity of IC 1613, NGC 3109 and WLM}

The HST-COS observations delivered an interesting by-product: the iron content of IC 1613 and WLM is similar or even larger than the $\sim 1 / 5 \mathrm{Fe}_{\odot}$ content of the SMC, superseding the $1 / 7 \mathrm{Z}_{\odot}$ value scaled from oxygen (Garcia et al. 2014; Bouret et al. 2015). Tautvaišienè et al. (2007) obtained a similar value from the analysis of three RSGs in IC 1613. Meanwhile Hosek et al. (2014) reported an $\sim 0.21 \mathrm{Fe}_{\odot}$ iron abundance for the massive stars of NGC 3109. The updated $\gtrsim 0.2 \mathrm{Fe} \odot$ content of IC 1613, NGC 3109 and WLM also helps to alleviate the problem of strong winds, as iron is the main driver of mass loss (e.g. Vink et al. 2001) and a larger wind-momentum is expected.

These results reflect the known fact that the ratio of $\alpha$-elements to Fe depends on the chemical evolution of galaxies, and that oxygen is not always a good proxy for metallicity. Unfortunately for the subject of this work, it also implies that the regime of massive stars with poorer metal-content than the $S M C\left(\lesssim 0.1 \mathrm{Fe}_{\odot}\right)$ remains unexplored.

\section{A promising $1 / 10 Z \odot$ galaxy: Sextans A}

Located 1.3Mpc away, Sextans A is potentially the most iron-poor galaxy of the Local Group. Hosek et al. (2014) compiled iron and $\alpha$-element abundances of nearby irregular galaxies determined from blue supergiants, thus probing the present-day metallicity while avoiding the calibration issues of nebular studies: Sextans A stands out as the most ironpoor with $[\mathrm{Fe} / \mathrm{H}] \lesssim-1.0$ and $[\alpha / \mathrm{Fe}] \sim 0$.

Camacho et al. (2016) recently confirmed the first OB-stars in Sextans A (see Table 1) with low-resolution long-slit spectroscopy performed at the GTC. The data enabled 
the first determination of the stellar parameters but yielded no information on the wind or abundances (as expected). The identified stars are not very massive, $M_{i n i} \sim 20-40 \mathrm{M}_{\odot}$, and 4-6Myr old. The youngest ones concentrate on the main galactic-scale over-densities of neutral hydrogen, similarly to IC 1613 (Garcia et al. 2010), although no very-early spectral types were detected. However, the most massive stars of Sextans A may be gasand dust-enshrouded within the HII shells, and out of reach to the depth of the observing program.

The UV spectra of some Sextans A OB-stars were obtained by pushing HST-COS to its limits (Fig.4). The stars display wind profiles that are weak, but strong enough to set constraints on the wind properties (Garcia et al. in prep). The continuum depletion caused by $\mathrm{Fe}$ and $\mathrm{Ni}$ transitions is almost negligible, specially when comparing with IC 1613 or SMC stars (see Fig. 4), therefore confirming the poor Fe content of the stars.

Sextans A may become the next sub-SMC metallicity standard and important observational effort is being devised to mine this extremely interesting galaxy. Stay tuned for GTC-OSIRIS multi-object spectroscopic observations (Camacho et al. in prep).

\section{Summary and outlook: prospects for the E-ELT and LUVOIR}

The analysis of stars in the $1 / 7 \mathrm{O}_{\odot}$ galaxies IC 1613, NGC 3109 and WLM has not revealed significant differences with SMC stars to date. This could be partly due to the very similar iron content of the four galaxies. The IC 1613, NGC 3109 and WLM stars open the window to study the effects of non-solar abundance patterns in the evolution and winds of massive stars, although such task would require much larger samples of stars.

The number of confirmed OBA stars in the true sub-SMC metallicity Sextans A is still scarce. The large distance to the galaxy, $1.3 \mathrm{Mpc}$, makes observations challenging. In addition, internal extinction at the expected sites for OB stars may be non-negligible since the youngest populations often co-exist with ionized and neutral gas, and dust. Deeper observations are needed.

In fact, progress in the characterization of sub-SMC metallicity massive stars requires efforts in 2 steps. Firstly deep, low-resolution (R 700-2000), wide-field spectroscopic observations are key to pierce through gas and dust, reach sufficiently weak stars, and enlarge the samples. In this respect, the high multiplexing capabilities of the secondgeneration spectrographs on $8-10 \mathrm{~m}$ telescopes will be crucial. We can expect significant contributions with MEGARA at the GTC (Gil de Paz et al. 2016) and MUSE at the VLT (Henault et al. 2003) in the near future.

Secondly stellar properties, wind parameters and abundances will be derived from quantitative spectroscopic analysis. The required ultraviolet observations and medium resolution ( $\mathrm{R} \sim 5000)$ optical spectroscopy again meet the problem of reaching stars as faint as $\mathrm{V} \sim 20-21$ and extinction, critical to the UV. It is important to stress that UV observations (optimally in the $950-1800 \AA$ range) are a necessary companion to optical spectroscopy. This range hosts both the diagnostics for the terminal velocity of OB stars and key lines to constrain shocks in the wind (Garcia \& Bianchi 2004) and micro-/macroclumping (Sundqvist et al. 2011).

We are pushing current observing facilities to the limit. Not only larger collecting surfaces are needed, but also source confusion cripples studies of individual objects in interesting sites farther than Sextans A.

Beyond the Local Group, I Zw18 (18.2Mpc Aloisi et al. 2007) is the most metal poor galaxy known to sustain significant star formation. With $1 / 32 \mathrm{Z}_{\odot} \quad$ (Vílchez \& IglesiasPáramo 1998), it may become the reference to study star formation in the first galaxies 
of the Universe and it is the ideal site to test $1 / 50 \mathrm{Z}_{\odot}$ stellar tracks. In fact, I Zw18 exhibits intense HeII4686 emission (Kehrig et al. 2015) that could be caused by Szécsi et al. (2015)'s TWUINs, very hot and massive stars experiencing CHE. However, I Zw18 is about 10 times farther than Sextans A and resolving its stellar population escapes current facilities.

The field will experience a tremendous boost with the arrival of two great astronomical facilities in the near/mid-term future. Multi-object spectrographs at the E-ELT (e.g. MOSAIC) will be able to reach main-sequence O-stars almost as far as 4Mpc, and resolve intricate environments like 30 Dor out to $1.5 \mathrm{Mpc}$. The second key facility is NASA's likely next flagship mission LUVOIR, a $12 \mathrm{~m}$ space telescope with UV, optical and IR capabilities. The observatory would be sensitive to unprecedentedly faint UV sources per se, but it will also host a multi-object spectrograph for UV observations (LUMOS) that will enable to observe entire extragalactic OB-associations in one shot. Both large facilities will be able to target individual, luminous stars in I Zw18. The synergies between E-ELT and LUVOIR will surely revolutionize the studies of truly metal-poor massive stars.

\section{Acknowledgements}

M. Garcia acknowledges funding by the Spanish MINECO via grants FIS2012-39162C06-01, ESP2013-47809-C3-1-R and ESP2015-65597-C4-1-R.

\section{References}

Aloisi, A., Clementini, G., Tosi, M., et al. 2007, ApJL, 667, L151

Azzopardi, M., Lequeux, J., \& Maeder, A. 1988, A\& $A, 189,34$

Bouret, J.-C., Lanz, T., Hillier, D. J., et al. 2015, MNRAS, 449, 1545

Bresolin, F., Pietrzyński, G., Urbaneja, M. A., et al. 2006, ApJ, 648, 1007

Bresolin, F., Urbaneja, M. A., Gieren, W., Pietrzyński, G., \& Kudritzki, R.-P. 2007, ApJ, 671, 2028

Brott, I., de Mink, S. E., Cantiello, M., et al. 2011a, A\&A, 530, A115

Camacho, I., Garcia, M., Herrero, A., \& Simón-Díaz, S. 2016, A\&A, 585, A82

Evans, C. J., Bresolin, F., Urbaneja, M. A., et al. 2007, ApJ, 659, 1198

Fullerton, A. W., Massa, D. L., \& Prinja, R. K. 2006, ApJ, 637, 1025

Garcia, M. \& Bianchi, L. 2004, ApJ, 606, 497

Garcia, M., Herrero, A., Castro, N., Corral, L., \& Rosenberg, A. 2010, A\&A, 523, A23

Garcia, M. \& Herrero, A. 2013, A\&3A, 551, A74

Garcia, M., Herrero, A., Najarro, F., Lennon, D. J., \& Alejandro Urbaneja, M. 2014, ApJ, 788, 64

Gil de Paz, A., Gallego, J., Carrasco, E., et al. 2016, Multi-Object Spectroscopy in the Next Decade: Big Questions, Large Surveys, and Wide Fields, 507, 103

Henault, F., Bacon, R., Bonneville, C., et al. 2003, SPIE, 4841, 1096

Herrero, A., Garcia, M., Uytterhoeven, K., et al. 2010, A\&A, 513, A70

Herrero, A., Garcia, M., Puls, J., et al. 2012, A\&A, 543, A85

Hosek, M. W., Jr., Kudritzki, R.-P., Bresolin, F., et al. 2014, ApJ, 785, 151

Kehrig, C., Vílchez, J. M., Pérez-Montero, E., et al. 2015, ApJL, 801, L28

Kudritzki, R.-P. \& Puls, J. 2000, ARA\&A, 38, 613

Kudritzki, R. P. 2002, ApJ, 577, 389

Leitherer, C., et al. 1992, ApJ, 401, 596

Leitherer, C., Leão, J. R. S., Heckman, T. M., et al. 2001, ApJ, 550, 724

Lozinskaya, T. A., Arkhipova, V. P., Moiseev, A. V., \& Afanas'Ev, V. L. 2002, Astronomy Reports, 46, 16

Madau, P. \& Dickinson, M. 2014, ARA\&A, 52, 415 
Mandel, I. \& de Mink, S. E. 2016, MNRAS, 458, 2634

Martins, F., Schaerer, D., \& Hillier, D. J. 2005, A\&SA, 436, 1049

Martins, F., et al. 2013, A\& $A$, 554, A23

Massey, P., Zangari, A. M., Morrell, N. I., et al. 2009, ApJ, 692, 618

Meynet, G., Maeder, A., Schaller, G., et al. 1994,A\&AS, 103, 97

Mokiem, M. R., de Koter, A., Vink, J. S., et al. 2007, A\&A, 473, 603

Sundqvist, J. O., Puls, J., Feldmeier, A., \& Owocki, S. P. 2011, A\&AA, 528, A64

Szécsi, D., Langer, N., Yoon, S.-C., et al. 2015, A\&A, 581, A15

Tautvaišienè, G., Geisler, D., Wallerstein, G., et al. 2007, AJ, 134, 2318

Tramper, F., Sana, H., de Koter, A., \& Kaper, L. 2011, ApJL, 741, L8

Tramper, F., Gräfener, G., Hartoog, O. E., et al. 2013, A\&SA, 559, A72

Tramper, F., Sana, H., de Koter, A., Kaper, L., \& Ramírez-Agudelo, O. H. 2014, A\&\&A, 572, AA36

Tramper, F., Straal, S. M., Sanyal, D., et al. 2015, A\&A, 581, A110

Trundle, C., Dufton, P. L., Hunter, I., et al. 2007, $A \& A$, 471, 625

Vílchez, J. M. \& Iglesias-Páramo, J. 1998, ApJ, 508, 248

Vink, J. S., et al. 2001, A\&\&A, 369, 574

\section{Discussion}

